\title{
Beyond PCMHs and Accountable Care Organizations: Payment Reform That Encourages Customized Care
}

\author{
Carrie H. Colla, PhD and Elliott S. Fisher, MD, MPH \\ The Dartmouth Institute for Health Policy and Clinical Practice, Geisel School of Medicine, 35 Centerra Parkway, Lebanon.
}

J Gen Intern Med 29(10):1325-7

DOI: $10.1007 / \mathrm{s} 11606-014-2928-4$

(c) Society of General Internal Medicine 2014

Question: How is a kilowatt-hour of electricity like a visit to a physician?

Answer: Nobody wants either.

H ealth care, like electricity, is an instrumental good, a H means to an end. ${ }^{1}$ We don't want kilowatt hours; we want hot showers and cold beer. We don't want to trek to the physician's office, we want health. How markets are structured determines how people behave. Energy policies have begun to reward utility companies for keeping people warm at the least possible cost, for example, through home insulation programs. Similarly, payment reforms in health care have begun to move away from those that reward volume to alternative approaches intended to improve the value of care. Patient-centered medical homes (PCMHs) were an important step, as fee-for-service payment provided no direct support for many key functions of primary care. Accountable care organizations (ACOs) were another advance, as they encourage collaboration across providers to improve care and lower costs. As we approach the fifth anniversary of the passage of the Affordable Care Act, it is a good time to reflect on how we are doing in payment policy — and how we might do better.

The commentary by Edwards and colleagues in this issue focuses on the need to restructure payment for primary care, with a particular focus on how this might be accomplished within ACOs. ${ }^{2}$ Health plans usually support the PCMH model through additional fees to support non-visit aspects of primary care on top of fee-for-service payments that continue to reward face-to-face visits. The authors contrast the PCMH and ACO models in terms of organizational structure, payment models, scope of accountability, and hypothesized degree of integration of specialists and hospitals. They conclude by suggesting possible approaches to integrating the PCMH model in ACOs: health plans could include explicit payments to primary care providers in ACOs (as in the PCMH model) and the performance-based

Published online August 5, 2014 payment component of ACOs could be restructured to more strongly reward the achievement of primary care principles.

The commentary is an excellent reminder that how health care is organized and paid for is important. Both the PCMH and ACO models have strengths and limitations. The PCMH model provides additional resources to practices enabling them to more easily expand access. The limitations, however, are substantial: the payment model focuses on adherence to a set of structural criteria that have a variable evidence base; the model continues to rely on fee for service and thus emphasizes visit-based care, and the model leaves unaddressed the challenge of how primary care relates to specialists or hospitals (thus current attention to the medical neighborhood). ${ }^{3}$ At the same time, because of the strong consensus on the need to strengthen primary care in the US, medical home initiatives are proliferating - with a still limited evidence base on their effectiveness. ${ }^{4}$

The ACO model includes a commitment to ensuring high-quality primary care. But it differs in several important ways. The ACO model was intentionally designed to encourage the emergence of organizations that could be responsible for the full continuum of care and for meeting quality and cost targets for the patients they serve-while allowing diverse approaches to emerge that could include - or not-hospitals, specialists or nursing homes. While the PCMH and ACO models are being broadly adopted, we are still in a learning stage. PCMHs are prescriptive; they require investments in specific practice characteristics, while ACOs allow for experimentation and goal setting at an organizational level.

Our National Survey of ACOs reveals that in spite of concerns that hospitals would dominate ACO formation, the majority are physician led and ACOs may be closer to PCMHs and more focused on primary care than many realize. ${ }^{5}$ In $87 \%$ of ACOs, at least one member of the ACO has experience as a PCMH and $56 \%$ of ACOs with 
commercial contracts continue to receive per member, per month fees from at least one payer to support care coordination, as in PCMHs. A small proportion of ACOs are comprised solely of primary care clinicians (12\%), and in $28 \%$ of ACOs primary care clinicians make up more than three-quarters of the clinicians. Of the ACOs with specialists, on average there are over two primary care clinicians for each specialist clinician in an ACO. ${ }^{6}$ These data show that ACOs are heavily primary care focused.

Early evidence indicates ACOs almost universally meet their quality targets and that some savings are achieved. However, the current model is increasingly recognized to have serious limitations. The complete freedom to seek care from any provider allowed by claims-based attribution of patients to ACOs has made patient engagement challenging for ACOs. The lack of standardized performance measures across public and private payers (in sharp contrast to the standardization inherent in the PCMH model) is a substantial, and unnecessary, burden. The reliance on annual shared savings payments means that securing capital for the investment in care redesign and improvement is difficult. Finally, most providers are straddling traditional fee-forservice and new risk-based payment approaches, making full commitment to care redesign difficult. ${ }^{7}$

Both the ACO and PCMH reform concepts should be understood in a broader framework that could help guide clinicians and policy makers as they move forward. The opportunity to improve both models lies in serious attention to three areas: clarifying our aims; developing measures aligned with those aims; and a commitment to learning how measurement, payment and regulatory changes can help accelerate improvement.

Accountable for what? Customized care that meets patient's goals. Some people want warm showers, while others prefer them piping hot. Recognizing that individuals differ in their goals, preferences and values, a growing chorus of voices is arguing that health care should focus on what matters to patients. ${ }^{8-10}$ Victor Montori and colleagues make an elegant plea for "minimally disruptive medicine": an approach to care that aims to increase patient's capacity to meet their personal goals and explicitly recognizes that medical treatment requires work that can either steal from limited reserves of energy-or enhance individuals' capacity to achieve their goals. ${ }^{11}$ This movement acknowledges that health care is an instrumental good. We would do well to set aims for health care that are explicitly linked to identifying what matters to patients, helping them meet those goals and minimizing the burden that health care imposes. Both the PCMH and ACO models acknowledge the importance of "patient-centered care," but this term has become so vague and all encompassing that it is virtually meaningless. Policy makers and health systems should embrace an explicit commitment to being accountable for customizing care to help patients achieve their goals.

How would we know we're getting there? Measuring what matters. We are very unlikely to make real progress on meeting patients' goals and reducing the burden of illness and the burden of health care (that unnecessary visit) until we make progress on measurement. This may include explicit documentation of individual patient goals (e.g., pain relief, mobility, social engagement) in a context of patient understanding of what is possible and potential tradeoffs as called for by Reuben and Tinetti. ${ }^{10}$ An outcome-focused approach could plausibly be built on the foundation established by NIH's PROMIS initiative, which is developing health status and domain-specific non-proprietary instrument banks that can be efficiently administered through computeradaptive testing that markedly reduces respondent burden. These measures could be used to track improvement on the domains that patients identify as most important. This approach will take time, but should be pursued. In the meantime, measurement of whether patients' goals are being elicited and incorporated into care plans is easily within reach. For example, CollaboRATE is a three-item patient-reported measure that captures whether the provider has elicited what matters to the patient, helped them understand their current situation and incorporated their preferences in the care plan. ${ }^{12}$ Measuring and reporting what matters to patients (both quality and cost) and how well providers are doing at meeting patient goals - while keeping the burden of measurement low - will get us closer to customized care for each patient.

Accelerating the transition to customized care. A health care system focused on engaging, enabling and empowering patients to meet their personal goals and aspirations in ways that minimize the burden imposed by health care will require concerted effort. Research funders, such as the Patient Centered Outcomes Research Institute and the Center for Medicare and Medicaid Innovation, will need to invest in further development of efficient approaches to measuring how patients are doing on what matters to them, in testing how to incorporate these measures in clinical workflows and - most importantly - in evaluating how best to reform delivery and payment models to achieve the aim of customized care. In the near term, public and private payers should consider implementing measures of decision quality in their upcoming revisions to the quality measures required for value-based payment initiatives that are already underway, from the pay-for-performance programs aimed at individual physicians to broader $\mathrm{PCMH}$ and $\mathrm{ACO}$ initiatives.

The current health care system, even within most patientcentered medical homes and accountable care organizations, remains largely focused on meeting professionally deter- 
mined aims in care models designed for the convenience of physicians - not patients. This is the equivalent of using kilowatt hours of electricity to produce cold showers and hot beer. We can do better.

Conflict of Interest: None

Corresponding Author: Elliott S. Fisher, MD, MPH; The Dartmouth Institute for Health Policy and Clinical Practice, Geisel School of Medicine, 35 Centerra Parkway, Lebanon NH 03755 (e-mail: elliott.s.fisher@dartmouth.edu).

\section{REFERENCES}

1. Berwick DM, Fisher ES, Lovins A. Negawatts and Negabeds. Huffington Post, January 29th, 2009.

2. Edwards ST, Abrams MK, Baron RJ, et al. Structuring payment to medical homes after the affordable care act. Journal of General Internal Medicine. 2014. doi:10.1007/s11606-014-2848-3.

3. Fisher ES. Building a medical neighborhood for the medical home. New England Journal of Medicine. 2008;359:1202-5.
4. Friedberg MW, Schneider EC, Rosenthal MB, Volpp KG, Werner RM. Association between participation in a multipayer medical home intervention and changes in quality, utilization, and costs of care. JAMA. 2014;311:815-25.

5. Colla CH, Lewis VA, Shortell SM, Fisher ES. First National Survey Of ACOs Finds Physicians Are Playing Strong Leadership And Ownership Roles. Health Aff (Millwood) Forthcoming.

6. Colla CH, Lewis VA, Shortell SM, Fisher ES. National Survey of Accountable Care Organizations. In: The Dartmouth Institute for Health Policy and Clinical Practice, ed. http://tdi.dartmouth.edu/initiatives/ accountable-care-organizations/research/NSACO; 2013.

7. Toussaint $\mathbf{J}$, Milstein A, Shortell $\mathbf{S}$. How the pioneer aco model needs to change: Lessons from its best-performing aco. JAMA. 2013;310:1341-2.

8. Bisognano M, Goodman E. Engaging patients and their loved ones in the ultimate conversation. Health Affairs. 2013;32:203-6.

9. Meyer GS, Nelson EC, Pryor DB, et al. More quality measures versus measuring what matters: a call for balance and parsimony. BMJ Quality \& Safety. 2012;21:964-8.

10. Reuben DB, Tinetti ME. Goal-oriented patient care-an alternative health outcomes paradigm. New England Journal of Medicine. 2012;366:777-9.

11. May C, Montori VM, Mair FS. We need minimally disruptive medicine. BMJ 2009;339.

12. Elwyn G, Barr PJ, Grande SW, Thompson R, Walsh T, Ozanne EM. Developing collaboRATE: a fast and frugal patient-reported measure of shared decision making in clinical encounters. Patient Education and Counseling. 2013;93:102-7. 